\title{
User Interface Input by Device Movement
}

\author{
Ryosuke Kokaji ${ }^{1}$, Takako Nonaka ${ }^{2}$, and Tomohiro Hase ${ }^{3}$ \\ ${ }^{1}$ Ryukoku University, Japan, t07m072@mail.ryukoku.ac.jp \\ ${ }^{2}$ Research Center for Information Communication Systems, Japan, \\ nonaka@rcics.hrc.ryukoku.ac.jp \\ ${ }^{3}$ Ryukoku University, Japan, hase@rins.ryukoku.ac.jp
}

\begin{abstract}
This paper develops a user interface to input by moving a terminal device. First, a concept of the portable viewer with magnifying glass-like operation already proposed and remaining problems are described. Next, a new method to detect the device movement using optical flow is proposed. Then, a prototype with an embedded 32-bit MPU is built to verify the proposed method. As a result, basic functions necessary for the user interface operation of the image viewer were implemented in the proposed system.
\end{abstract}

Keywords: Embedded system, optical flow, portable viewer

\section{Introduction}

Recently, with portable information terminal devices becoming increasingly sophisticated, the number of various terminals with a small display as an output interface has increases. However, the amount of information that can be presented on the small display screen is limited, and therefore the terminal cannot display all information at once. The general methods for accessing hidden information are as follows: grouping and stratifying menus, and moving between the groups and hierarchies, and cutting out a part of a virtual screen, and scrolling to a hidden area. However, the user interfaces (UIs) share no common standards, and each model has different operation methods. This obviously can lead to confusion and frustration among the first time users. In addition, miniaturization and lightening the portable terminals are now important requirements, which reduce the area and volume occupied by special input interfaces on the device.

The authors have considered intuitive operation methods that are independent of previous usage experiences and knowledge about computers. The authors proposed a new UI whose input and output $(\mathrm{I} / \mathrm{O})$ relation is similar to a magnifying glass (Matsuda, 2007). 
This paper proposes a new method for detecting the device movement to achieve the proposed magnifying glass-like UI, and its validity is verified using a prototype.

\section{Concept of the proposed UI}

Figure 1 shows a system schematic to illustrate how the proposed magnifying glass-like UI works with the portable image viewer.

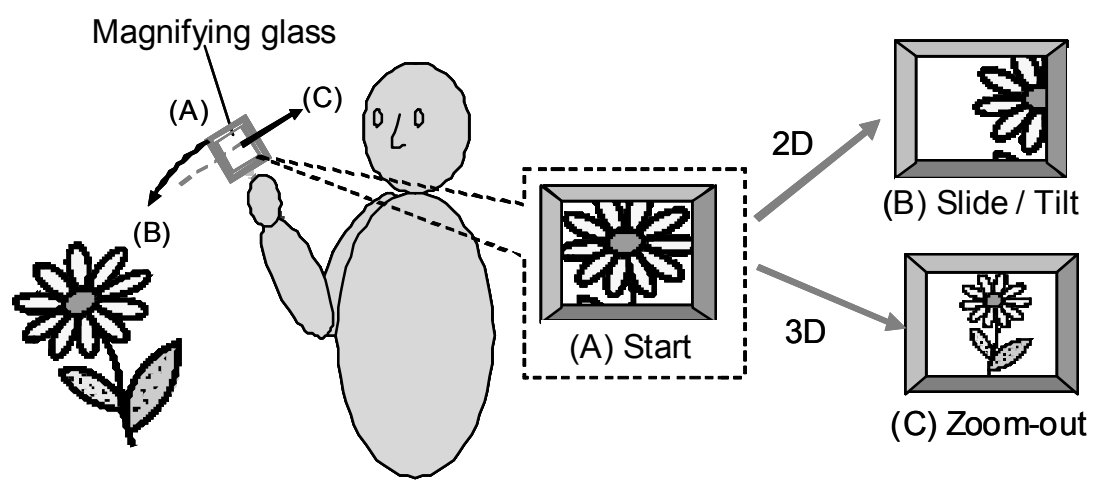

Figure 1 Concept of a portable image viewer with a magnifying glass-like UI

The proposed system aims at input operation without any pointing devices such as a mouse or a joystick. In addition, the relationship between the terminal movement and the output screen image on the small display is designed with the aim of achieving the relationship between the magnifying glass and an erect image formed by its convex lens. This will support those inexperienced with computer in understanding the operation method intuitively.

The required basic functions of the portable image viewer are as follows: rotation, movement, expansion and reduction of the image. It is therefore necessary to detect the gyration of the viewer body around three axes, as well as movement on three perpendicular spatial directions to achieve the proposed UI

\section{New method of movement detection of the terminal device}

There are many examples of detecting the terminal movement with acceleration sensors and gyroscopes. When detecting the terminal movement using these sensors, it is necessary to combine two or more sensors. 
On the other hand, portable information terminal units equipped with small cameras normally, e.g. cellular phones, are increasing recently. Then, this paper devises the method of movement detection of the terminal using miniature cameras embedded in the terminal units efficiently.

Table 1. Characteristics of movement detection using acceleration sensors, gyroscopes, and miniature cameras.

\begin{tabular}{l|l|l|l}
\hline \hline Sensors \& Devices & $\begin{array}{l}\text { Displacement } \\
\text { along an axis }\end{array}$ & $\begin{array}{l}\text { Rotation } \\
\text { about an axis }\end{array}$ & $\begin{array}{l}\text { Characteristics } \\
\text { \& Problems }\end{array}$ \\
\hline \hline Acceleration sensor & Detectable & $\begin{array}{l}\text { Undetectable } \\
\text { *Detectable inclined } \\
\text { angles by multiaxis }\end{array}$ & $\begin{array}{l}\text { Integration error } \\
\text { One sensor / axis }\end{array}$ \\
\hline $\begin{array}{l}\text { Gyroscope } \\
\text { (Rate sensor) }\end{array}$ & Undetectable & Detectable & $\begin{array}{l}\text { Integration error } \\
\text { One sensor / axis }\end{array}$ \\
\hline $\begin{array}{l}\text { Miniature camera } \\
\text { Optical flow }\end{array}$ & Detectable & Detectable & Processing load \\
\hline \hline
\end{tabular}

When operating the proposed system, the user is expected to stand face-to-face with the output screen. The distance between the viewer and the user presumed to have 20/20 vision is limited to the range from 10 to $60 \mathrm{~cm}$ because of the screen size and resolution.

Therefore, a miniature camera is placed near and on the same surface as a small display. Next, optical flow is detected by using images captured by the miniature camera. Finally, the terminal's movement is estimated using the detected motion vector, and used to operate the UI. Figure 2 illustrates the hardware structure of the proposed viewer and its usage.

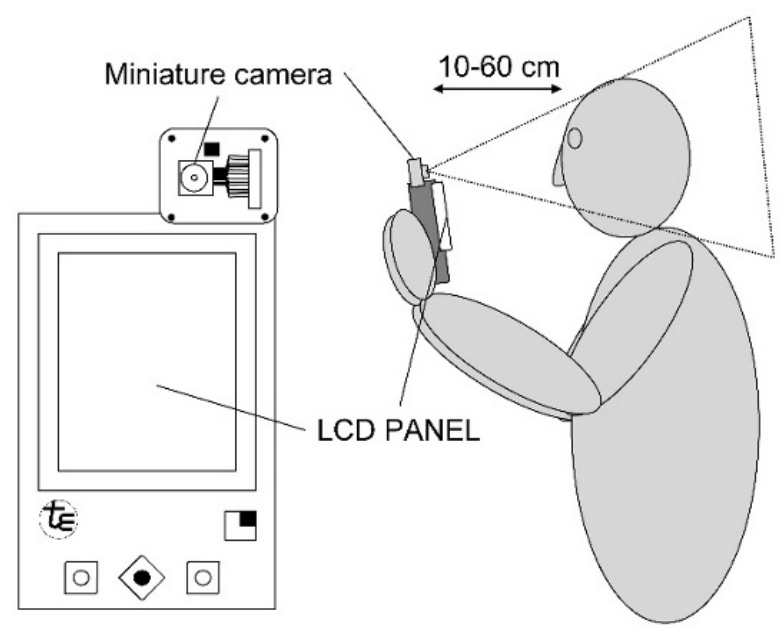

Figure $2 \mathrm{H} / \mathrm{W}$ structure of the proposed viewer. 
When operating the proposed system, it is expected that the user's face will always be captured by the miniature camera located on the terminal. Thus, optical flow centered on the face is detected, and the movement of the device is presumed. Fig. 3 shows the detection algorithm of the optical flow (Matsuda, 2006).

The detected motion vectors show the movements of the surroundings as seen from the terminal, and the terminal's movements are then estimated from the relative motion.

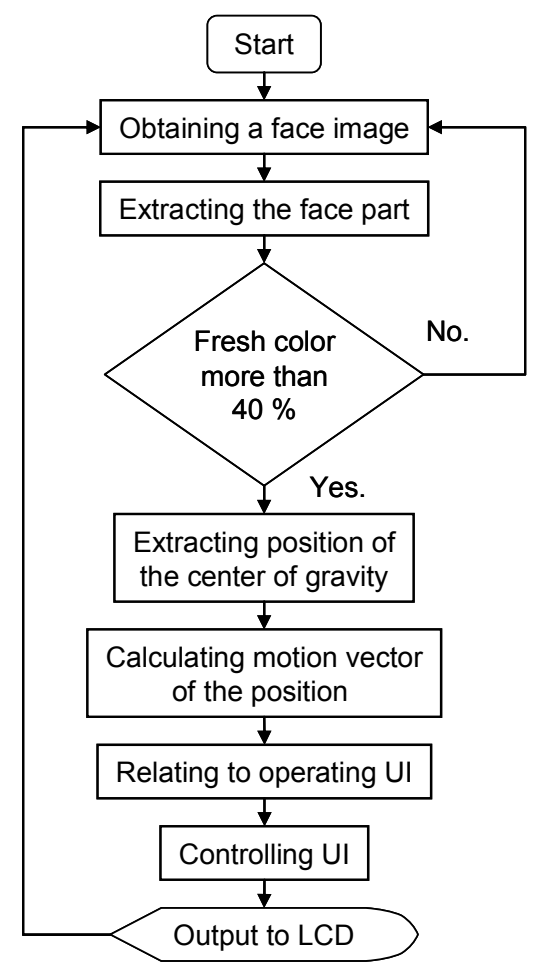

Figure 3 Detection algorithm of optical flow.

\section{Verification Experiments}

To verify the proposed method, a prototype with a miniature camera was built, based on a T-Engine embedded-device development standard platform. The prototype is shown in Figure 4 and the specifications are listed in Table 2.

The prototype operates on a 32-bit embedded MPU for consumer use, and its performance is reasonable for general portable terminals. 
During the course of the verification experiments, the prototype was held and operated face-to-face by the user with both hands. The terminal was moved up and down and right and left in order to move the output images on the screen.

Table 2 Specifications of the prototype system

\begin{tabular}{l|l}
\hline \hline Item & Specifications \\
\hline \hline MPU & M32700/300 MHz \\
\hline Memory & Flash 8 MB, SDRAM 32 MB \\
\hline OS & Linux / M32R (kernel2.6.14) \\
\hline LCD & $320 * 240$ (QVGA) \\
\hline Camera & $640 * 480($ VGA) \\
\hline \hline
\end{tabular}

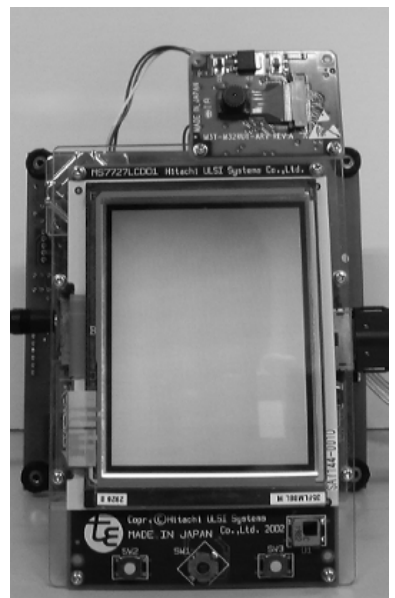

Figure 4 Photograph of the prototype.

As a result, when the terminal was moved up and down and/or right and left, the face movement was detected whenever user's face was captured by the camera, and the output image was then shifted using the motion vector.

\section{Conclusions}

Thus, the basic functions necessary for the UI operation of the image viewer were implemented in the proposed system. Furthermore, the desired magnifying glasslike operation was also achieved. It was possible to execute all computing and processing operations on a low specification system. Therefore, this proposed 
method could be easily implemented on a small portable terminal with a small display such as a cellular phone or a PDA.

\section{References}

Matsuda, M., Nonaka, T., Kokaji, R., Shimano, M., Hase, T.: Handy viewer with user interface according to device movement, Proc. ICCE 2007 on IEEE, pp. 319-320, Las Vegas (2007).

Matsuda, M., Nonaka, T., Hase, T.: An Input Method Using Hand Gestures for a Portable Remote Controller For Consumer Use, Proc. ICCE 2006 on IEEE, pp. 223-224, Las Vegas (2006). 